\title{
Numerical Simulation: Effects of Gas Flow and Heat Transfer on Polymer Deposition in a Plasma Dry Etcher
}

\author{
Junghoon Joo* \\ Department of Materials Science and Engineering, Kunsan National University, Gunsan, Jeonbuk 573-701, Korea
}

Received October 12, 2017; accepted November 14, 2017

\begin{abstract}
Polymer deposition pattern on the ceramic lid surface is analyzed by numerical modeling. Assumption was made that is affected by gas flow pattern from the horizontal and vertical nozzles, temperature profile from the fingerlike branches made of graphite and electrostatic potential effect. Calculated results showed gas flow dynamics is less relevant than two others. Temperature and electrostatic effects are likely determining the polymer deposition pattern based on our numerical simulation results.
\end{abstract}

Keywords: Plasma, Polymer, Deposition, Gas flow, Temperature, Electrostatic, ICP

\section{Introduction}

Dry etching of $\mathrm{Si}$ and $\mathrm{SiO}_{2}$ is done by competing reactions : deposition and etching on the film surfaces. Ions are bombarding the wafer surfaces with a few hundreds of $\mathrm{eV}$ by rf biasing through an electrostatic chuck. For $\mathrm{SiO}_{2}$ etching, high etch rate as a few $\mathrm{nm} / \mathrm{sec}$ is necessary as well as high selectivity, to substrate materials. Fluorocarbon gases are commonly used in dry etching of $\mathrm{SiO}_{2}: \mathrm{CF}_{4}, \mathrm{C}_{2} \mathrm{~F}_{6}$, $\mathrm{C}_{4} \mathrm{~F}_{6}$, and $\mathrm{C}_{4} \mathrm{~F}_{8} \mathrm{C} / \mathrm{F}$ ratio is a key factor to determine the etching characteristics. A dry etcher is composed of three key sections:a plasma source, a top lid and a lower chamber connected to a large capacity turbo molecular pump. In this study, we used a $300 \mathrm{~mm}$ wafer dry etcher made by AMAT (model name: DPS, decoupled plasma source). It has a dual turn inductively coupled plasma source with a current control circuit to give a uniform plasma profile over radial direction. ICP system has a thick insulator ceramic window between an antenna and a plasma. The process gap is between 100 and $200 \mathrm{~mm}$ as usual. IC chip yield strongly depends on the particle generation inside the etch chamber. $\mathrm{CF}_{\mathrm{x}}$ based polymer chemistry is generating thick polymer deposit on plasma exposed surfaces as the process time increases. Residual stress develops on some surfaces and thick polymer deposit finally falls off from the inner surfaces of the ceramic lid. Radial and azimuthal directional borders of the deposited polymer shows very distinct star like pattern. Correlation with gas injection nozzle, ICP antenna geometry and off-center pumping stack configuration are considered in this paper.

\section{Experiment and Simulation Setup}

DPS (decoupled plasma source) dry etcher can etch poly $\mathrm{Si}$ and metals. Each has specific gas injection nozzle configuration. Other parts of the system are very common in ICP etchers. Process gas pressures were adjusted to 10 $\sim 30$ mTorr by controlling MFCs (mass flow controller) to the tunable gas nozzle which has horizontal and vertical nozzles. Horizontal/Vertical flow rates are separately controlled by a ratio controller. The top ceramic lid is thermally controlled both by air cooling fans and a silicon rubber covered graphite heater. It has a circumferential element and radial branches. Additional heating source is practically the plasma. High density plasma region is formed between the inner turn and the outer turn antenna position at the lower surface of the ceramic top lid. Air cooling fans are mounted on the coil box top, slightly offcentered due to the rf matching box positioning. After operating ICP at $400 \mathrm{~W}$ for $40 \mathrm{~min}$, the thermal image of the air side surface of the lid was obtained by using IR (infra red) camera (Fluke Ti10). Numerical modeling was done by a commercial multi-physics software, CFDACE+. Three steps of modeling are:gas flow dynamics of plasma region, heat transfer analysis of coil box air, the lid heater and the electrostatic potential profile of the ceramic lid. All of the materials' characteristic constants, e.g. thermal conductivity, are referenced from the ESI's inherent material database. Numerical configuration of CFD-ACE+ and common structure of plasma simulation were published elsewhere.

*Corresponding author

E-mail:jhjoo@kunsan.ac.kr 


\section{Results and Discussion}

\section{1. $\mathrm{CF}_{\mathrm{x}}$ polymer deposition}

$\mathrm{CF}_{4}$ gas is a basic process gas in poly silicon and oxide etching. Plasma generated polymers are $\mathrm{Si} \mathrm{CF}_{\mathrm{x}}$ and $\mathrm{SiO}_{2} \mathrm{CF}_{\mathrm{x}}$. The remaining thickness of these polymers depends on the ion sputtering rate and thermal temperature of the surface. Plasma density profile is assumed to be uniform between the inner and the outer coils in the numerical model. Dust particle collision induced sputtering effect was reported in a reference. In this study, the polymer deposition pattern (Fig. 1) is the target to be analyzed and simulated by numerical models. The downwards direction of the photo is the TMP (turbo molecular pump) direction. So the arguments in the ref. 6 (dust particle collision from the fast running blades of the TMP. Slightly blurred fashion of the polymer deposition profile may be an evidence of the ref. 6's arguments. It was not possible in the current simulation by CFD-ACE+. It has a macro particle simulation module. So it would be possible to analyze by a combined model including all four effects: gas flow, macro particle, heat transfer and

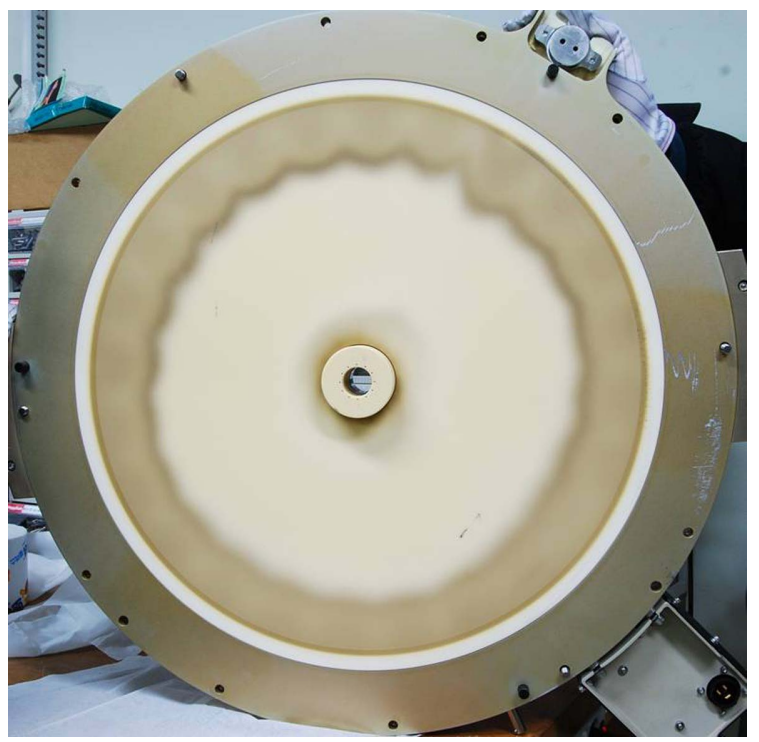

Figure 1. Polymer deposition pattern on the lower surface of the ceramic lid/.

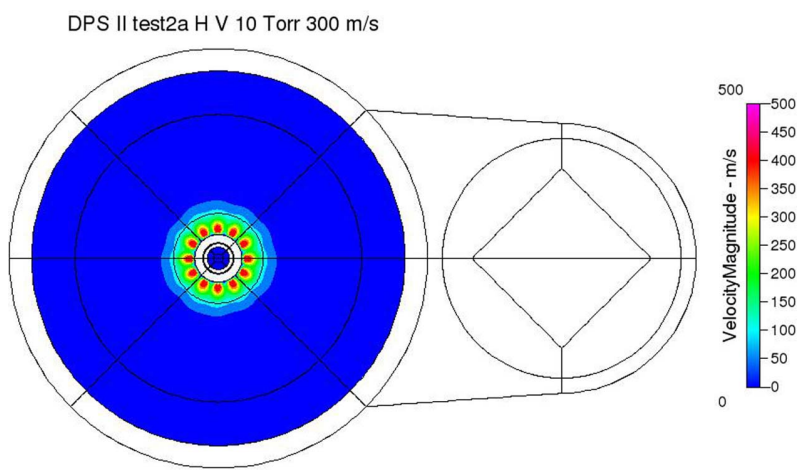

Figure 2. Computer simulated gas flow dynamic pattern of 12 hole tunable gas nozzle. electrostatic profile in the future.

\section{Gas flow effects}

The tunable gas nozzle (TGN) has 12 horizontal and vertical nozzles. In Fig. 2, the gas flow pattern near at the nozzles is showing that the velocity profile would get more uniform than the polymer deposition pattern as shown in Fig. 1. In the vertical section of the CFD result showed the locality of gas flow pattern invoked from the nozzle position and direction would not exist over a few $\mathrm{cm}$ away from the nozzle surfaces. In Fig. 4, the gas flow pattern is getting affected by off-center TMP position.

Based on this computational results, gas flow effects may not be a crucial source for the polymer deposition pattern.

\section{Temperature effects}

Polymer thickness profile is a deposition process. It is affected by the surface temperature. In order to control it, the ceramic lid can be heated by two sources : plasma and finger like top heater. In this model, plasma is assumed to be generated by two coils : inner and outer turns. If the rf driving current is in the same direction, most of rf power is deposited between the inner and outer turn area. The lid edge area's temperature is determined by three sources: ceramic lid, plasma and aluminum chamber body. Chamber body is about $20 \mathrm{~mm}$ thick and cooled by liquid coolant. The ceramic lid is floated by an O-ring from the chamber.

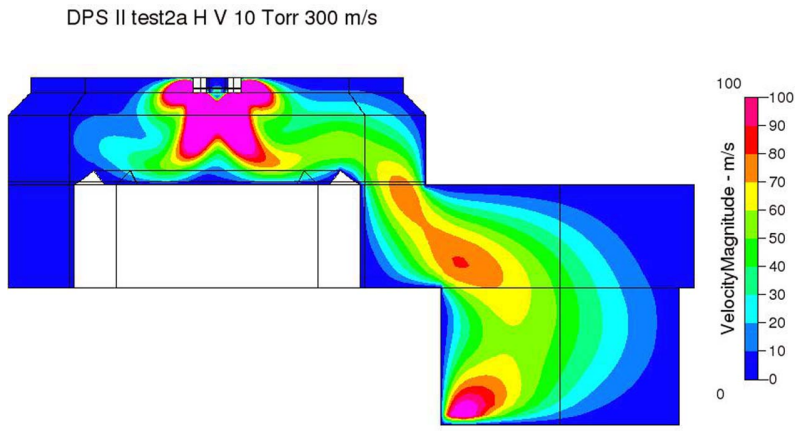

Figure 3. Vertical cut profile of the simulated gas flow dynamic pattern showing horizontal and vertical gas injection extends only within a few $\mathrm{cm}$.

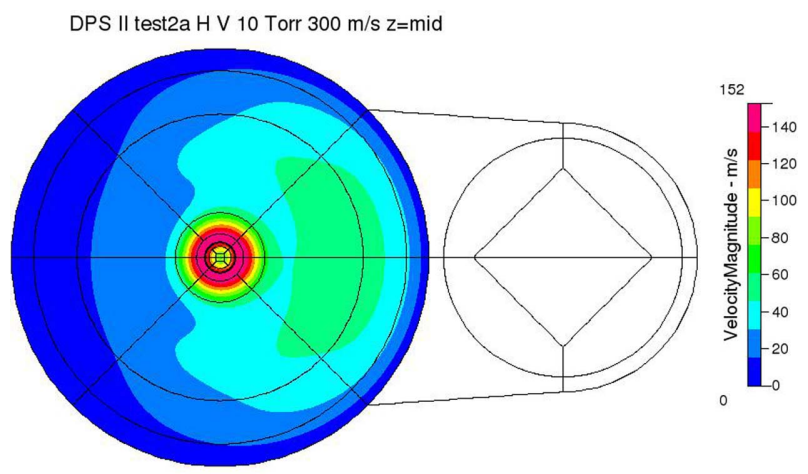

Figure 4. Horizontal cut profile of the CFD results showing skewed pattern toward the turbo molecular pump. 


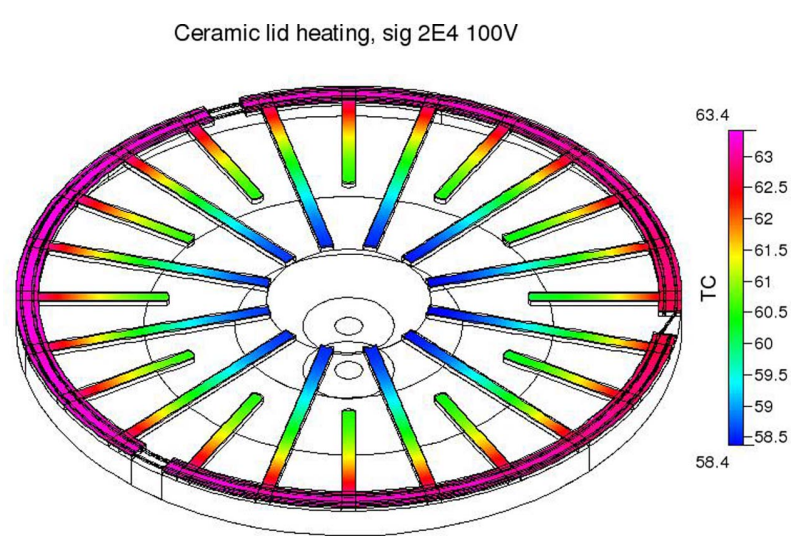

Figure 5. Temperature profile of the radial branch top surfaces.

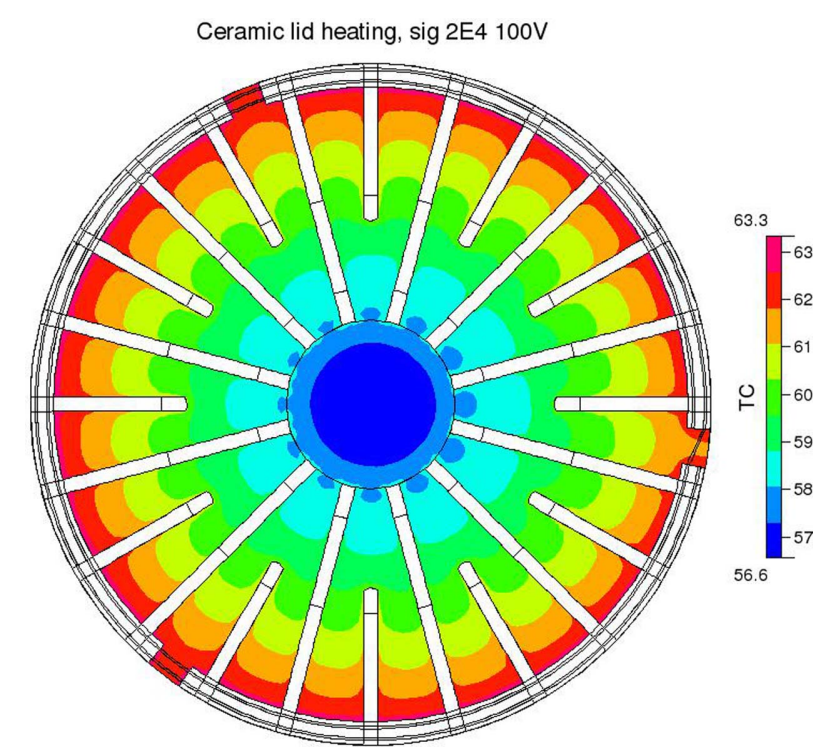

Figure 6. Temperature profile of the ceramic lid surfaces.

DPS II coil box cooling $1 \mathrm{~m} / \mathrm{s}$

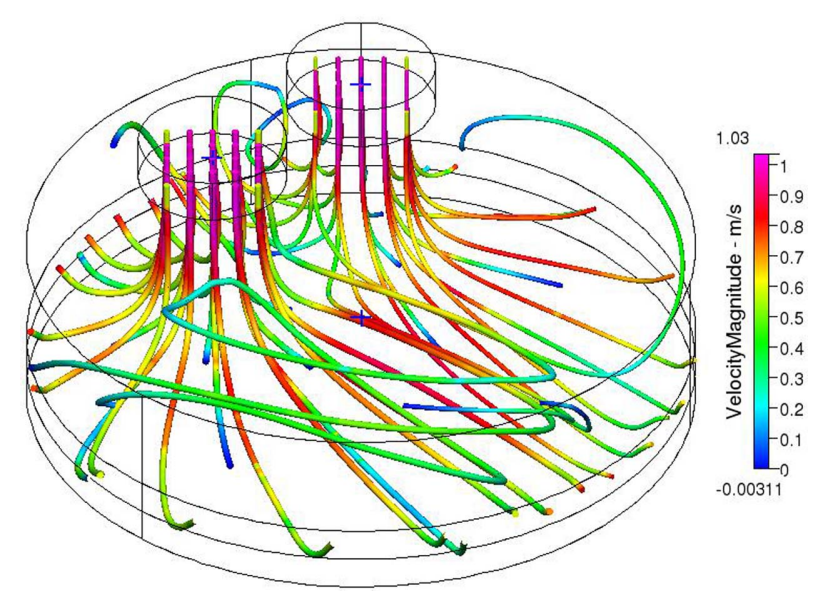

Figure 7. Cooling air flow traces from two fans.

Two cooling fans are blowing ambient air down to the surface of the ceramic lid. Due to the rf matching box position, fans are located to the left side of it. Air ventilation holes are on the side wall of the rf antenna

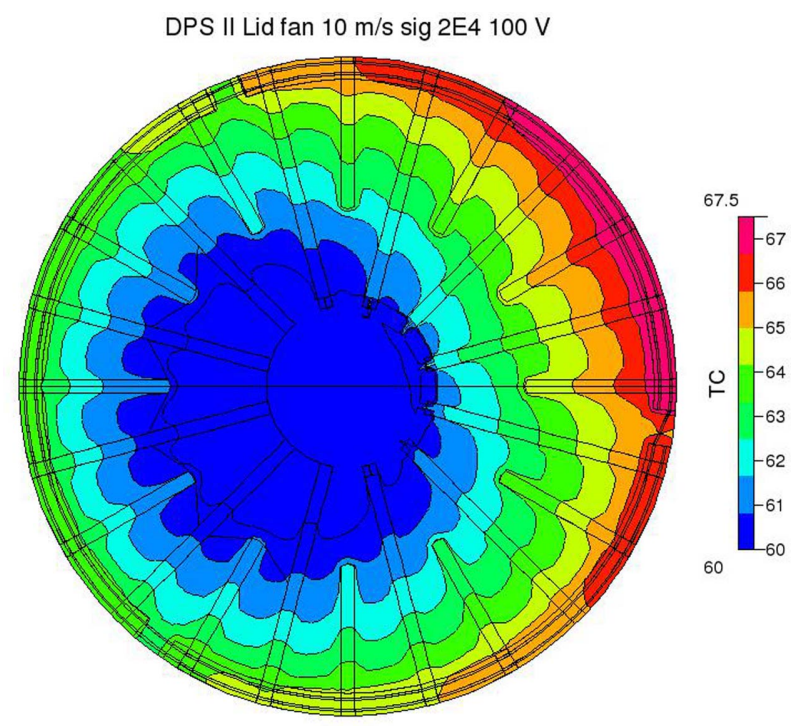

Figure 8. Temperature profile of the ceramic lid surfaces including cooling fan effects.

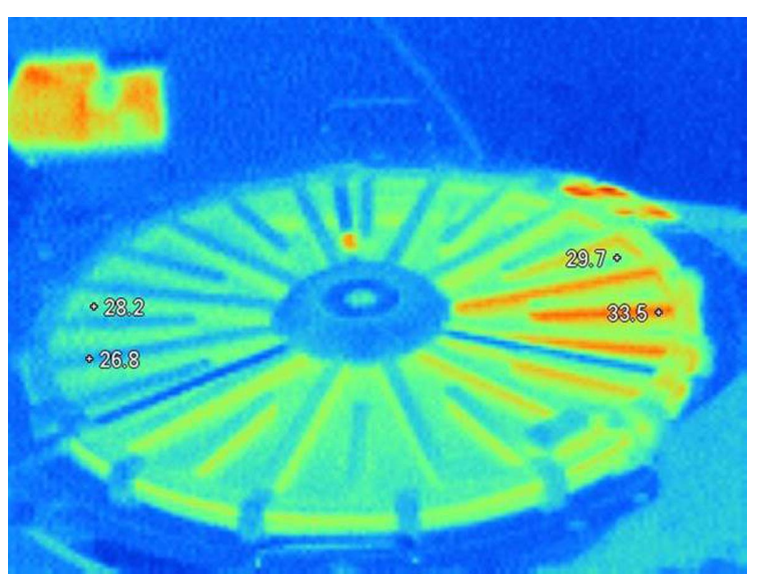

Figure 9. Thermal imaging profile taken after $40 \mathrm{~min}$ operation of ICP at $300 \mathrm{~W}$.

section. So the ceramic lid surfaces are cooled unevenly. The graphite heaters have long and short branches covered by silicone rubber layers to shield from cooling air. Thermal imaging camera showed this unbalanced temperature distribution of the ceramic lid surface.

In Fig. 5 and Fig. 6, the calculated temperature profile of the branches of heating elements and the ceramic lid surface are shown. Each one is showing the sawtooth profile of the optical image of Fig. 1. Two cooling fans are modeled as vertical air inlet condition, showing traces in Fig. 7. Due to this cooling effects, the temperature profile of the ceramic lid surface is skewed to right side in Fig. 8. It is very similar to the thermal imaging by an IR camera in Fig. 9.

However, the lower surface of the ceramic lid is heated by ion bombardment from plasma. In Fig. 10, the temperature profile of the lid's surface without considering ion bombardment is shown. The edge temperature is higher than inside region, e.g. TGN. If we model the ICP's heating effect as a constant heat input area like donut, the 


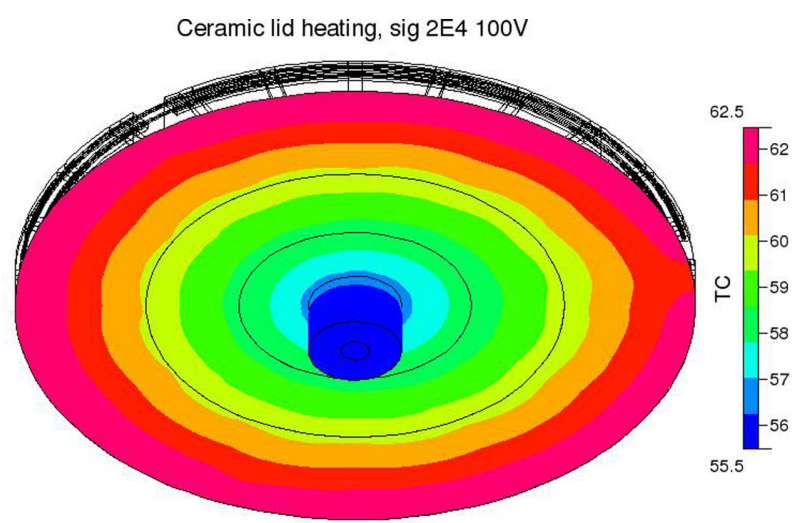

Figure 10. Temperature profile of the ceramic lid's lower surfaces.

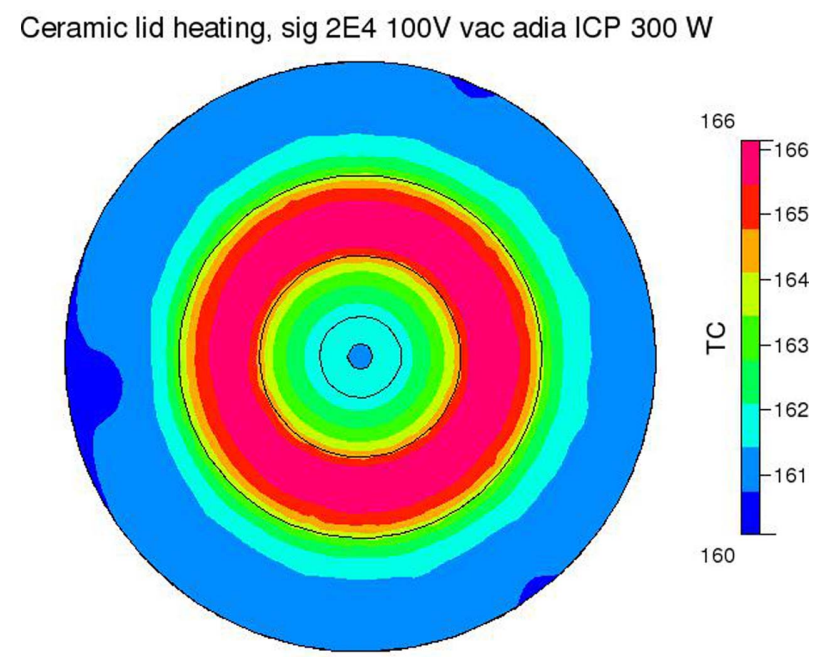

Figure 11. Temperature profile of the ceramic lid's lower surfaces including ICP heating effect.

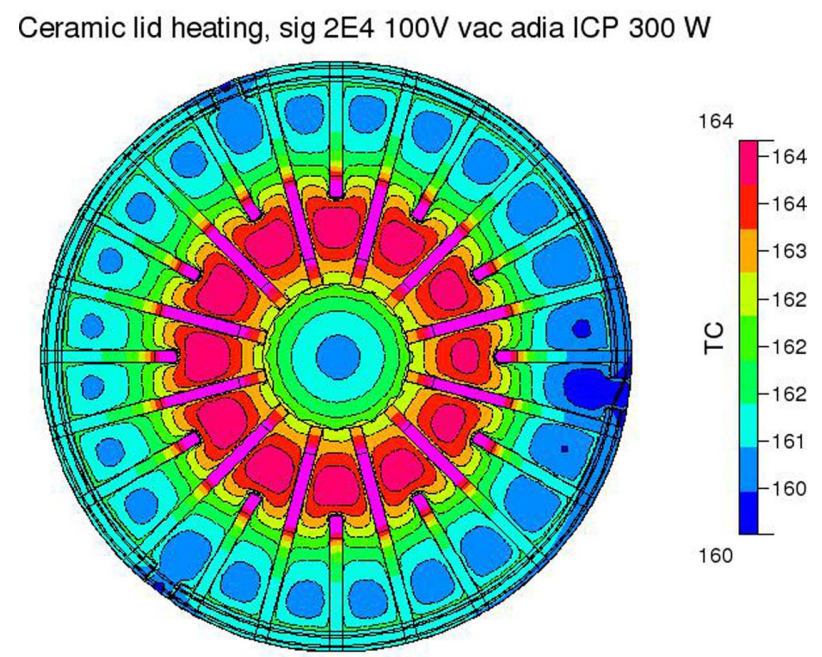

Figure 12. Temperature profile of the ceramic lid's air side surfaces including ICP heating effect.

temperature profile changes to that in Fig. 11. At the same condition, the lid surface to the case air would get temperature profile as shown in Fig. 12. Temporary conclusion is that the polymer deposition profile cannot be explained by heat transfer effect alone.
Electric potential profile at mid level of ceramic lid

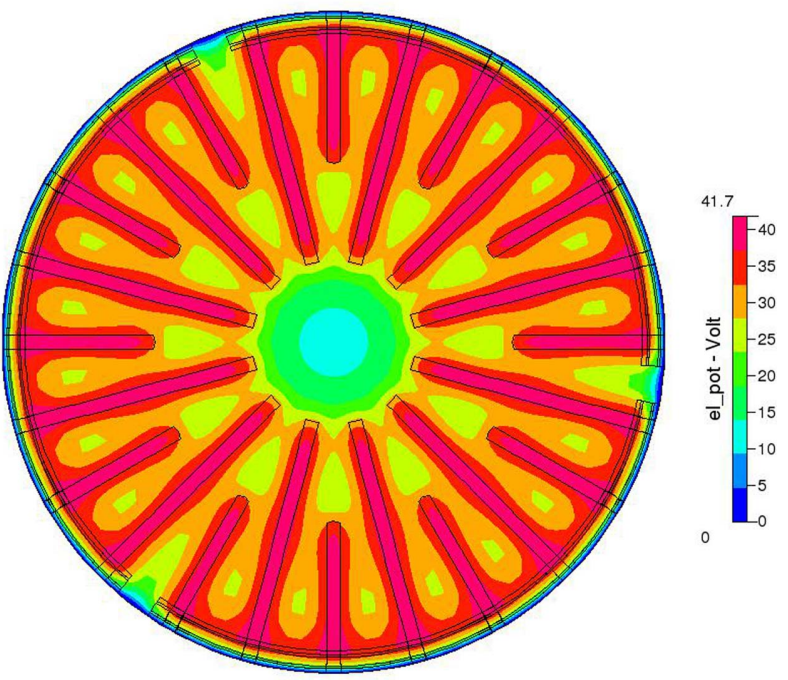

Figure 13. Electric potential profile of the ceramic lid's air side surfaces.

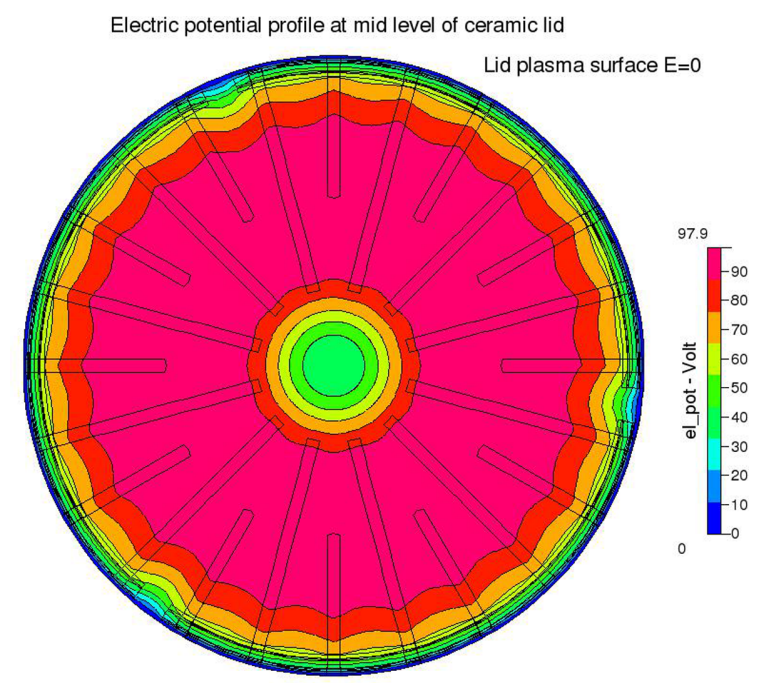

Figure 14. Electric potential profile of the ceramic lid's plasma side surfaces.

\section{Capacitive coupling effects}

Radial branch style heating elements are made of a Ni$\mathrm{Cr}$ heating wire and a graphite. Both are electrically conductive. ICP rf antenna radiates electromagnetic fields downwards to the chamber. Any material with finite electrical conductivity would interfere this rf EM fields. This would invoke two effects:confinement of rf power absorption and electrostatic surface potential change. Polymer deposition on the lower surface of the ceramic lid is affected by ion bombardment from plasma. Heating elements are powered by balanced $60 \mathrm{~Hz}$ AC. On the average, it would be considered as electrically grounded. Combined electric potential from rf antenna and grounded heating elements would determine the surface potential of the ceramic lid exposed to the plasma. In order to investigate this argument, a numerical model was built. The graphite heating elements and wires are assigned as 
$100 \mathrm{~V}$ and the ceramic lid (alumina ceramic, relative dielectric constant 9.7). The ceramic lid surface toward plasma is assumed to have $10 \mathrm{~V}$ of floating potential or zero tangential electric field. The first case showed a profile which is affected by the shape of heating elements made of graphite as shown in Fig. 13. The later case (Fig. 14) showed very similar to the observed pattern of polymer deposition on the inner surfaces of the ceramic lid.

\section{Conclusions}

From numerical modeling, the polymer deposition pattern on the inner surface of the ceramic lid of a AMAT DPS II dry poly Si etcher, was affected by gas flow, temperature profile of the ceramic lid and ion bombardment which is determined by electric potential difference between the lid surface and the nearby plasma.
Among these causes, the temperature and the electric potential are the most crucial causes of this polymer deposition pattern.

\section{Acknowledgements}

This research is supported by WC300 research project under contract no 10046957 by the Korean Ministry of Trade, Industry and Energy.

\section{Reference}

[1] Junghoon Joo, Appl. Sci. Conv. Eng. Vol. 23, No. 4, (2014) 169.

[2] Junghoon Joo, J. Kor. Inst. Surf. Eng. Vol. 45, No. 4, (2012) 174.

[3] H. Kim, W. Yang, and J. Joo, J. Appl. Phys. Vol. 118 (2014) 043304-1.

[4] J. Joo, Thin Solid Films, Vol 519 (2011) 6892.

[5] S. Lee, J. Kor. Vac. Sci. Technol. Vol. 18, No. 3 (2009) 176.

[6] H. Kobayashi, Jpn. J. Appl. Phys. Vol50 (2011) 08JE01. 